\title{
The Riesz-Kolmogorov theorem on metric spaces
}

\author{
Przemystaw Górka and Anna Macios
}




\title{
THE RIESZ-KOLMOGOROV THEOREM ON METRIC SPACES
}

\author{
PRZEMYSŁAW GÓRKA AND ANNA MACIOS
}

Received 24 September, 2013

\begin{abstract}
We study precompact sets in $L^{p}(X, \mu)$, where $(X, \mu)$ is a metric measure space. Using Vitali and maximal function theorems we establish a full characterization of such sets.
\end{abstract}

2010 Mathematics Subject Classification: 28C99; 46B50; 46E30

Keywords: metric measure spaces, precompact sets, Riesz-Kolmogorov theorem

\section{INTRODUCTION}

Compact sets are important object of analysis' research because of their important properties and huge applications. Therefore, question about full characterization of compact or precompact sets (sets such that their closure is compact) in different spaces is very important. Riesz-Kolmogorov theorem gives necessary and sufficient conditions for precompactness of subset of $L^{p}\left(\mathbb{R}^{n}\right)$ (see $\left.[6,10]\right)$. This theorem plays a fundamental role in analysis. Especially, it is frequently used in the theory of function spaces, e.g. Sobolev and Besov spaces.

The goal of the paper is to give a characterization of precompact sets in $L^{p}(X, \mu)$, where $(X, \mu)$ is a metric space with doubling measure, i.e positive Borel measure satisfying condition

$$
0<\mu(B(x, 2 r)) \leq C \mu(B(x, r))<\infty
$$

for all $x \in X, r>0$ and some constant $C>0$ called doubling constant. We shall denote the average of locally integrable function $f$ over the measurable set $A$ in the following manner

$$
(f)_{A}=\frac{1}{\mu(A)} \int_{A} f d \mu .
$$

In this note we would like to present the following observation.

The first author enjoyed the support by the European Union in the framework of European Social Fund through the Warsaw University of Technology Development Programme.

(C) 2014 Miskolc University Press 
Theorem 1. Let $\mu$ be a doubling measure such that

$$
h(r):=\inf \{\mu(B(x, r)): x \in X\}>0 \text { for each } r>0
$$

and assume that $1<p<\infty$. Let $x_{0} \in X$, then the subset $\mathcal{F}$ of $L^{p}(X, \mu)$ is relatively compact in $L^{p}(X, \mu)$ if and only if the following conditions are satisfied:

$$
\begin{array}{rr}
\lim _{R \rightarrow \infty} \int_{X \backslash B\left(x_{0}, R\right)}|f(x)|^{p} d \mu(x)=0, & \text { uniformly for } f \in \mathcal{F}, \\
\lim _{r \rightarrow 0} \int_{X}\left|f(x)-(f)_{B(x, r)}\right|^{p} d \mu(x)=0 & \text { uniformly for } f \in \mathcal{F} .
\end{array}
$$

We can find a bit similar theorem in [5], i.e. sufficient condition for precompactness of subset of $L^{p}(X, \mu)$ for $1 \leq p<\infty$, where $(X, \mu)$ is a metric space with finite measure. The proof of our theorem is obtained by appropriate modifications in the proof of theorem mentioned above [5]. We apply Vitali convergence theorem. Moreover, Lebesgue differentiation theorem as well as Hardy-Littlewood maximal function theorem will be used.

Finally, let us mention about some generalizations of the Riesz-Kolmogorov theorem. For instance, Weil [11] showed the compactness theorem in $L^{p}(G)$, where $G$ is a locally compact group. Pego [8] (see also [2]) formulated Kolmogorov theorem for $p=2$ in terms of the Fourier transform. There also exists a characterization of relatively compact subsets of general Banach spaces [9].

\section{Proof of THEOREM}

First of all we assume that conditions (1.1)-(1.3) hold. Let $f_{n}$ be a sequence of elements from $\mathcal{F}$. We shall prove that $f_{n}$ has a subsequence converging in $L^{p}(X, \mu)$. Since the set $\mathscr{F}$ is bounded and the space $L^{p}(X, \mu)$ is reflexive, we may assume that $f_{n}$ converges weakly to some $f \in L^{p}(X, \mu)$. We can represent the space $X$ in the following manner

$$
X=\bigcup_{n=1}^{\infty} B\left(x_{0}, n\right) .
$$

Now, we shall show that for each $n$

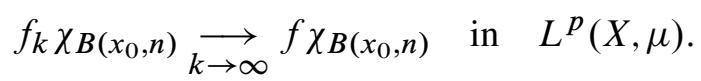

We apply Vitali convergence theorem (see e.g. [7]). We first prove that the sequence $f_{k} \chi_{B\left(x_{0}, n\right)}$ is $p$-equi-integrable. For this purpose we fix $\epsilon>0$. By (1.3), there exists $r$ such that for each $k$

$$
\int_{X}\left|f_{k}(x)-\left(f_{k}\right)_{B(x, r)}\right|^{p} d \mu(x) \leq \frac{\varepsilon}{2} .
$$


Moreover, we fix a measurable set $A$ such that

$$
\mu(A) \leq\left(\frac{\varepsilon}{2 \sup _{k \in \mathbb{N}}\left\|f_{k}\right\|_{L^{p}(X)}}\right)^{p} h(r) .
$$

Then, using Minkowski and Hölder inequality we get

$$
\begin{aligned}
& \left(\int_{A}\left|f_{k} \chi_{B\left(x_{0}, n\right)}(x)\right|^{p} d \mu(x)\right)^{\frac{1}{p}} \leq\left(\int_{A}\left|f_{k}(x)\right|^{p} d \mu(x)\right)^{\frac{1}{p}} \\
& \leq\left(\int_{A}\left|f_{k}(x)-\left(f_{k}\right)_{B(x, r)}\right|^{p} d \mu(x)\right)^{\frac{1}{p}}+\left(\int_{A}\left|\left(f_{k}\right)_{B(x, r)}\right|^{p} d \mu(x)\right)^{\frac{1}{p}} \\
& \leq \frac{\varepsilon}{2}+\left(\int_{A} \frac{1}{(\mu(B(x, r)))^{p}}\left|\int_{B(x, r)} f_{k}(y) d \mu(y)\right|^{p} d \mu(x)\right)^{\frac{1}{p}} \\
& \leq \frac{\varepsilon}{2}+\left(\int_{A} \frac{1}{\mu(B(x, r))} \int_{B(x, r)}\left|f_{k}(y)\right|^{p} d \mu(y) d \mu(x)\right)^{\frac{1}{p}} \\
& \leq \frac{\varepsilon}{2}+\left(\frac{\mu(A)}{h(r)}\right)^{\frac{1}{p}} \sup _{k \in \mathbb{N}}\left\|f_{k}\right\|_{L^{p}(X)}<\varepsilon .
\end{aligned}
$$

This ends the proof of $p$-equi-integrability.

Our next claim is that $f_{k} \chi_{B\left(x_{0}, n\right)} \underset{k \rightarrow \infty}{\stackrel{\mu}{\longrightarrow}} f \chi_{B\left(x_{0}, n\right)}$ in measure. Let us take $\varepsilon>0$. We note

$$
\begin{aligned}
& \left\{x \in X:\left|f_{k} \chi_{B\left(x_{0}, n\right)}(x)-f \chi_{B\left(x_{0}, n\right)}(x)\right|>\varepsilon\right\}=\left\{x \in B\left(x_{0}, n\right):\left|f_{k}(x)-f(x)\right|>\varepsilon\right\} \\
& \subset\left\{x \in B\left(x_{0}, n\right):\left|f_{k}(x)-\left(f_{k}\right)_{B(x, r)}\right|>\frac{\varepsilon}{3}\right\} \\
& \quad \cup\left\{x \in B\left(x_{0}, n\right):\left|\left(f_{k}\right)_{B(x, r)}-(f)_{B(x, r)}\right|>\frac{\varepsilon}{3}\right\} \\
& \quad \cup\left\{x \in B\left(x_{0}, n\right):\left|f(x)-(f)_{B(x, r)}\right|>\frac{\varepsilon}{3}\right\} \\
& =A_{1} \cup A_{2} \cup A_{3} .
\end{aligned}
$$

It suffices to prove that for each $\delta>0$, there exists $K$ such that for each $k \geq K$, the inequality

$$
\mu\left(A_{1}\right)+\mu\left(A_{2}\right)+\mu\left(A_{3}\right)<\delta
$$


holds. Using Markov's inequality we get

$$
\begin{aligned}
\mu\left(A_{1}\right) & \leq\left(\frac{3}{\varepsilon}\right)^{p} \int_{B\left(x_{0}, n\right)}\left|f_{k}(x)-\left(f_{k}\right)_{B(x, r)}\right|^{p} d \mu(x) \\
& \leq\left(\frac{3}{\varepsilon}\right)^{p} \int_{X}\left|f_{k}(x)-\left(f_{k}\right)_{B(x, r)}\right|^{p} d \mu(x) .
\end{aligned}
$$

Therefore, by assumption (1.3), there exists $r_{0}$ such that for each $r \leq r_{0}$ we get

$$
\mu\left(A_{1}\right)<\frac{\delta}{3} .
$$

Next, we consider the set $A_{2}$. We conclude from $f_{k} \underset{k \rightarrow \infty}{\stackrel{w}{\longrightarrow}} f$ that for each $x \in$ $B\left(x_{0}, n\right)$ and $r>0$

$$
\left(f_{k}\right)_{B(x, r)} \rightarrow(f)_{B(x, r)} .
$$

This gives $\left(f_{k}\right)_{B(x, r)} \stackrel{\mu}{\rightarrow}(f)_{B(x, r)}$ in measure on $B\left(x_{0}, n\right)$. By the definition, there exists $K(r)$ such that for each $k \geq K(r)$ the inequality $\mu\left(A_{2}\right)<\frac{\delta}{3}$ holds.

It remains to consider the set $A_{3}$. Using Markov's inequality we have

$$
\mu\left(A_{3}\right) \leq D_{r}:=\left(\frac{3}{\varepsilon}\right)^{p} \int_{B\left(x_{0}, n\right)}\left|f(x)-(f)_{B(x, r)}\right|^{p} d \mu(x) .
$$

Thanks to Lebesgue differentiation theorem (see [4]) we get that $\lim _{r \rightarrow 0} f_{B(x, r)}=f(x)$ a.e. on $X$. Direct calculations leads us to the following estimates

$$
\begin{aligned}
\left|f(x)-f_{B(x, r)}\right|^{p} & \leq\left(|f(x)|+\mid(f)_{B(x, r) \mid}\right)^{p} \leq 2^{p-1}\left(|f(x)|^{p}+\left|(f)_{B(x, r)}\right|^{p}\right) \\
& \leq 2^{p-1}\left(|f(x)|^{p}+|\mathcal{M}(f)(x)|^{p}\right),
\end{aligned}
$$

where $\mathcal{M}(f)$ is a maximal function of $f$. By virtue of the Hardy-Littlewood maximal function theorem (see [4]) we have $\|\mathcal{M}(f)\|_{L^{p}(X, \mu)} \leq C(p)\|f\|_{L^{p}(X, \mu)}$, where $p>$ 1. Therefore, since $|f|^{p}$ is integrable, the Lebesgue dominated convergence theorem gives

$$
\lim _{r \rightarrow 0} D_{r}=\left(\frac{3}{\varepsilon}\right)^{p} \int_{B\left(x_{0}, n\right)} \lim _{r \rightarrow 0}\left|f(x)-(f)_{B(x, r)}\right|^{p} d \mu(x)=0
$$

and hence there exists $r_{1}$, such that for $r \leq r_{1}$ we have

$$
\left(\frac{3}{\varepsilon}\right)^{p} \int_{B\left(x_{0}, n\right)}\left|f(x)-(f)_{B(x, r)}\right|^{p} d \mu(x)<\frac{\delta}{3} .
$$

Finally, taking $\tilde{r}=\min \left\{r_{0}, r_{1}\right\}$, we obtain the inequality

$$
\mu\left(\left\{x \in X:\left|f_{k} \chi_{B\left(x_{0}, n\right)}(x)-f \chi_{B\left(x_{0}, n\right)}(x)\right|>\varepsilon\right\}\right)<\delta
$$


for each $k \geq K(\tilde{r})$. Now, we show that

$$
f_{k} \underset{k \rightarrow \infty}{\longrightarrow} f \text { in } L^{p}(X, \mu) .
$$

Let us fix $\varepsilon>0$. Since $f \in L^{p}(X, \mu)$ and by (1.2), there exists $N$ such that

$$
\int_{X \backslash B\left(x_{0}, N\right)}\left|f_{k}(x)-f(x)\right|^{p} d \mu(x) \leq \frac{\varepsilon}{2} .
$$

Hence,

$$
\begin{aligned}
\left\|f_{k}-f\right\|_{L^{p}(X, \mu)}^{p}= & \int_{X}\left|f_{k} \chi_{B\left(x_{0}, N\right)}(x)-f \chi_{B\left(x_{0}, N\right)}(x)\right|^{p} d \mu(x) \\
& +\int_{X \backslash B\left(x_{0}, N\right)}\left|f_{k}(x)-f(x)\right|^{p} d \mu(x) \leq \varepsilon .
\end{aligned}
$$

This ends the proof of relatively compactness of $\mathcal{F}$.

Now, we shall show the converse. We assume that the family $\mathcal{F}$ is relatively compact. Hence, the boundedness is straightforward. To establish condition (1.2), let us fix $\varepsilon>0$ and let $\mathcal{U}=\left\{U_{1}, U_{2}, \ldots, U_{n}\right\}$ be an $\varepsilon$-cover of $\mathcal{F}$. For each $k=1, \ldots, n$ we can select $g_{k} \in U_{k}$ and $R_{k}>0$ such that

$$
\int_{X \backslash B\left(x_{0}, R_{k}\right)}\left|g_{k}(x)\right|^{p} d \mu(x)<\varepsilon .
$$

Let us fix $f \in \mathscr{F}$. Since $U$ is an $\varepsilon$-cover of $\mathcal{F}$, there exists $1 \leq k \leq n$ such that $f \in U_{k}$. Thus,

$$
\int_{X}\left|f(x)-g_{k}(x)\right|^{p} d \mu(x)<\varepsilon
$$

and for $R=\max \left\{R_{i}: 1 \leq i \leq n\right\}$ we get

$$
\begin{aligned}
\int_{X \backslash B\left(x_{0}, R\right)}|f(x)|^{p} d \mu(x) \leq & 2^{p-1} \int_{X \backslash B\left(x_{0}, R\right)}\left|f(x)-g_{k}(x)\right|^{p} d \mu(x) \\
& +2^{p-1} \int_{X \backslash B\left(x_{0}, R\right)}\left|g_{k}(x)\right|^{p} d \mu(x)<2^{p} \varepsilon .
\end{aligned}
$$

So condition (1.2) holds.

Using $g_{k}$ choosen as above, we obtain:

$$
\begin{aligned}
\int_{X}\left|f(x)-(f)_{B(x, r)}\right|^{p} d \mu(x) \leq & 2^{p-1} \int_{X}\left|f(x)-g_{k}(x)\right|^{p} d \mu(x) \\
& +2^{2(p-1)} \int_{X}\left|g_{k}(x)-\left(g_{k}\right)_{B(x, r)}\right|^{p} d \mu(x) \\
& +2^{2(p-1)} \int_{X}\left|\left(g_{k}\right)_{B(x, r)}-(f)_{B(x, r)}\right|^{p} d \mu(x) \\
= & 2^{p-1} I_{1}+2^{2(p-1)} I_{2}+2^{2(p-1)} I_{3} .
\end{aligned}
$$


We easily get $I_{1}<\varepsilon$. Now, using the Hardy-Littlewood maximal function theorem we obtain

$$
\begin{aligned}
I_{3} & =\int_{X}\left|\frac{1}{\mu(B(x, r))} \int_{B(x, r)}\left(g_{k}(y)-f(y)\right) d \mu(y)\right|^{p} d \mu(x) \\
& \leq \int_{X}\left|\mathcal{M}\left(g_{k}-f\right)(x)\right|^{p} d \mu(x) \leq C(p)^{p} \int_{X}\left|g_{k}(x)-f(x)\right|^{p} d \mu(x) \leq C(p)^{p} \varepsilon .
\end{aligned}
$$

Now it remains to prove that $I_{2}$ is arbitrary small. By Lebesgue differentiation theorem we obtain that

$$
\lim _{r \rightarrow 0}\left|g_{k}(x)-\left(g_{k}\right)_{B(x, r)}\right|=0, \quad \text { a.e. on } X
$$

Moreover,

$$
\left|g_{k}(x)-\left(g_{k}\right)_{B(x, r)}\right|^{p} \leq 2^{p-1}\left(\left|g_{k}(x)\right|^{p}+\left|\mathcal{M}\left(g_{k}\right)(x)\right|^{p}\right) .
$$

Since $\left|g_{k}\right|^{p}$ and $\left|\mathcal{M}\left(g_{k}\right)\right|^{p}$ are integrable, we can apply Lebesgue dominated convergence theorem. Thus,

$$
I_{2}=\int_{X}\left|g_{k}(x)-\left(g_{k}\right)_{B(x, r)}\right|^{p} d \mu(x)<\varepsilon
$$

and the proof is complete.

Let us remark that if $\mu$ is doubling and $\operatorname{diam} X<\infty$ or $\mu$ is continuous with respect to the metric $\rho$ (see [1,3]) and $X$ is compact, then for each $r>0, h(r)$ is positive.

\section{ACKNOWLEDGEMENT}

The authors would like to thank the anonymous referee for careful reading of the paper, correcting errors and pointing out places where the exposition is not entirely clear.

\section{REFERENCES}

[1] M. Gaczkowski and P. Górka, "Harmonic functions on metric measure spaces: Convergence and compactness," Potential Anal., vol. 31, pp. 203-214, 2009.

[2] P. Górka, "Pego theorem on locally compact abelian groups," J. Algebra Appl., vol. 13, 2014.

[3] P. Górka, "Campanato theorem on metric measure spaces," Ann. Acad. Sci. Fenn., Math., vol. 34, no. 2, pp. 523-528, 2009.

[4] J. Heinonen, Lectures on Analysis on Metric Spaces. Universitext, 2001.

[5] A. Kałamajska, "On compactness of embedding for sobolev spaces defined on metric spaces," Ann. Acad. Sci. Fenn., vol. 24, pp. 123-132, 1999.

[6] A. N. Kolmogorov, "Über kompaktheit der funktionenmengen bei der konvergenz im mittel," Machr. Ges. Wiss. Göttingen, vol. 9, pp. 60-63, 1931.

[7] G. Leoni, A first course in Sobolev spaces. Graduate Studies in Mathematics, AMS, 2009.

[8] R. L. Pego, "Compactness in $l^{2}$ and the fourier transform," Proc. Am. Math. Soc., vol. 95, pp. 252-254, 1985.

[9] R. S. Phillips, “On linear transforms,” Trans. Am. Math. Soc., vol. 48, pp. 516-541, 1940. 
[10] M. Riesz, "Sur les ensembles compacts de foncions sommables," Acta Szeged Sect. Math., vol. 6, pp. 136-142, 1933.

[11] A. Weil, L'intégration dans les groupes topologiques et ses applications. Hermann et Cie., Paris, 1940.

Authors' addresses

Przemysław Górka

Warsaw University of Technology, Department of Mathematics and Information Sciences, Ul. Koszykowa 75, 00-662 Warsaw, Poland

E-mail address: P.Gorka@mini.pw.edu.pl

Anna Macios

Warsaw University of Technology, Department of Mathematics and Information Sciences, Ul. Koszykowa 75, 00-662 Warsaw, Poland 